\title{
Comparative genomic analysis of Mycobacterium intracellulare: implications for clinical taxonomic classification in pulmonary Mycobacterium avium- intracellulare complex disease
}

Yoshitaka Tateishi ${ }^{1 *}$, Yuriko Ozeki ${ }^{1}$, Akihito Nishiyama ${ }^{1}$, Mari Miki ${ }^{2}$, Ryoji Maekura ${ }^{2,3}$, Yukari Fukushima ${ }^{4}$, Chie Nakajima ${ }^{4,5}$, Yasuhiko Suzuki ${ }^{4,5}$ and Sohkichi Matsumoto ${ }^{1,6}$

\begin{abstract}
Background: Mycobacterium intracellulare is a representative etiological agent of emerging pulmonary M. aviumintracellulare complex disease in the industrialized countries worldwide. The recent genome sequencing of clinical strains isolated from pulmonary M. avium-intracellulare complex disease has provided insight into the genomic characteristics of pathogenic mycobacteria, especially for M. avium; however, the genomic characteristics of $M$. intracellulare remain to be elucidated.

Results: In this study, we performed comparative genomic analysis of $55 \mathrm{M}$. intracellulare and related strains such as M. paraintracellulare (MP), M. indicus pranii (MIP) and M. yonogonense. Based on the average nucleotide identity, the clinical M. intracellulare strains were phylogenetically grouped in two clusters: (1) the typical M. intracellulare (TMI) group, including ATCC13950 and virulent M.i.27 and M.i.198 that we previously reported, and (2) the MP-MIP group. The alignment of the genomic regions was mostly preserved between groups. Plasmids were identified between groups and subgroups, including a plasmid common among some strains of the M.i.27 subgroup. Several genomic regions including those encoding factors involved in lipid metabolism (e.g., fadE3, fadE33), transporters (e.g., mce3), and type VII secretion system (genes of ESX-2 system) were shown to be hypermutated in the clinical strains. M. intracellulare was shown to be pan-genomic at the species and subspecies levels. The mce genes were specific to particular subspecies, suggesting that these genes may be helpful in discriminating virulence phenotypes between subspecies.
\end{abstract}

* Correspondence: y-tateishi@med.niigata-u.ac.jp

'Department of Bacteriology, Graduate School of Medical and Dental Sciences, Niigata University, Niigata, Japan

Full list of author information is available at the end of the article

(c) The Author(s). 2021 Open Access This article is licensed under a Creative Commons Attribution 4.0 International License, which permits use, sharing, adaptation, distribution and reproduction in any medium or format, as long as you give appropriate credit to the original author(s) and the source, provide a link to the Creative Commons licence, and indicate if changes were made. The images or other third party material in this article are included in the article's Creative Commons licence, unless indicated otherwise in a credit line to the material. If material is not included in the article's Creative Commons licence and your intended use is not permitted by statutory regulation or exceeds the permitted use, you will need to obtain permission directly from the copyright holder. To view a copy of this licence, visit http://creativecommons.org/licenses/by/4.0/ The Creative Commons Public Domain Dedication waiver (http://creativecommons.org/publicdomain/zero/1.0/) applies to the data made available in this article, unless otherwise stated in a credit line to the data. 
(Continued from previous page)

Conclusions: Our data suggest that genomic diversity among M. intracellulare, M. paraintracellulare, M. indicus pranii and $M$. yonogonense remains at the subspecies or genovar levels and does not reach the species level. Genetic components such as mce genes revealed by the comparative genomic analysis could be the novel focus for further insight into the mechanism of human pathogenesis for M. intracellulare and related strains.

Keywords: Mycobacterium intracellulare, Mycobacterium paraintracellulare, Mycobacterium indicus pranii, Comparative genomics, Mammalian cell entry genes

\section{Background}

Mycobacteria have successfully adapted to the human host environment as represented by the worldwide prevalence of tuberculosis and nontuberculous mycobacteriosis [1]. Mycobacterium avium and Mycobacterium intracellulare (MAC) are major causative agents of pulmonary nontuberculous mycobacterial disease in Europe, the US, and Asia, including Japan [2-5]. The identification of genetic differences between species and strains provides basic information for investigating the cause of differential epidemiology, virulence, and pathogenesis.

Recent advances in genome sequencing technology have enabled the comparison of clinically-isolated strains at the whole genome level and this has revealed that some mycobacterial species have open pan-genomes [69]. In $M$. avium, the genomic diversity is classified into several subspecies, including subspecies avium, hominissuis, paratuberculosis, and silvaticum [10]. M. avium subsp. avium causes avian tuberculosis in birds and other domestic animals such as pigs and cattle [11]. M. avium subsp. hominissuis causes pulmonary infections, cervical lymphadenitis, soft tissue infections and disseminated infections in humans and porcine [11]. M. avium subsp. paratuberculosis causes Johne's disease in ruminants and non-ruminant domestic animals (pigs, dogs, horses, cats, etc.), free ranging animals (rabbits, bears, rats, etc.) and non-human primates [12]. M. avium subsp. silvaticum causes tuberculosis-like disease in wood pigeons, cranes and deer [12]. Strains belonging to some clusters have been suggested to possess potential genetic factors that account for their clinical pathogenesis [9].

In $M$. intracellulare, the comparative genomic approach has not been reported for typing strains to date; nevertheless, new relevant species and subspecies have been proposed by multi-locus sequencing typing of a single strain, i.e., $M$. paraintracellulare (MP) [13], $M$. yongonense [14] and M. indicus pranii (MIP) [15, 16]. However, the grouping of $M$. intracellulare and its related strains is confusing in the NCBI genome database. As for February 2021, M. yongonense and MIP are registered as an independent subspecies of $M$. intracellulare, i.e. M. intracellulare subsp. yongonense and M. intracellulare subsp. intracellulare. And curiously, only one MIP strain, MTCC9506 belongs to M. intracellulare subsp. intracellulare on the NCBI genome database, in spite of a dozen of $M$. intracellulare strains (including the type strain ATCC13950) registered in a category of $M$. intracellulare species without designation of their subspecies. In addition, MP, whose reference strain was originally reported as $M$. intracellulare MOTT64 [17], is registered as an independent species in the NCBI genome database. The actual grouping of these strains, along with a large number of clinically-isolated strains, remains to be elucidated. According to some reports from South Korea, about 4 and 3\% of strains diagnosed as $M$. intracellulare by a commercial hybridization assay of $r p o B$ gene have been identified as MIP and M. yongonense by sequence-based typing analyses, respectively [18]. Although typing of strains based on the sequence of housekeeping genes is a quick and effective method for classifying clinical strains, the method of species verification at the single strain level needs scientific verification by comparative genomic analysis, as proposed by the comprehensive taxonomic analysis of nontuberculous mycobacteria [19, 20].

We have reported the virulence of clinical $M$. intracellulare strains (strains M.i.198 and M.i.27) in comparison with clinical $M$. avium strains [21]. We also identified a 50 -kb region of a prophage in the hypervirulent strain M.i.198 [22]. These data prompted us to investigate in more depth the genomic landscape at the multiple strain level. To understand the genomic similarity and diversity between clinically-isolated $M$. intracellulare strains, and previously-reported $M$. intracellulare and related species and subspecies, we performed comparative genomic analysis of $M$. intracellulare for 55 strains including 31 clinical strains (including M.i.198 and M.i.27) isolated from cases of pulmonary $M$. intracellulare disease. We clarified the classification of clinical $M$. intracellulare strains into two major groups: the typical $M$. intracellulare (TMI) group and the M. paraintracellulare - M. indicus pranii (MP-MIP) group. The genomic difference was not significant enough that species differentiation between the strains enrolled in this study was possible by means of the genomic alignment and the nucleotide identity. Therefore, we propose new insights into the clinical taxonomic classification of $M$. intracellulare and related strains. 


\section{Results}

Identification of the species of clinical strains and general genomic features

The clinical strains were assigned to species based on the best match strain among the NCBI reference strains (Table 1). In total, 17, 3, 5, and 6 strains were assigned to $M$. intracellulare ATCC13950, $M$. intracellulare MOTT-02, MP, and MIP, respectively. The percentage of mapped regions was high, ranging from 85 to $100 \%$. These data suggested that MP and MIP comprised approximately one-third of clinical strains diagnosed as $M$. intracellulare. No clinical strains were identified as $M$. yongonense.

\section{Phylogenetic analysis based on whole genome comparisons}

The strains enrolled in this study were grouped into two major groups: the typical $M$. intracellulare (TMI) group and the $M$. paraintracellulare-M. indicus pranii (MPMIP) group (Fig. 1, Fig. S1). In the TMI group, there were three major subgroups including ATCC13950, M.i.27 and M.i.198 as representative strains, and several minor subgroups including M007, M002, M014, M013, M018, M024 and M010. The subgroups of ATCC13950 included M005 and M016. The subgroup of M.i.27 included M009, M008, M028, M025, M006, M023 and M026. The subgroup of M.i.198 included 4 previouslyregistered strains including MOTT-02, FLAC01363, FLAC0181 and FLAC0204 and our clinical strains including M022 and M004.

Some strains classified in the MP-MIP group, such as M017 (MP designated by the best match strain in the NCBI database) and M003 (MIP designated by the best match strain in the NCBI database), were shown to be phylogenetically closely related to previously reported M. paraintracellulare strains (MOTT64, KCTC29084, and JCM30622) and M. indicus pranii MTCC9506, respectively. However, the majority of strains belonging to the MP-MIP group were classified into several subgroups that were closely related to MP and MIP species. With the exception of some parts of the genome registered in the NCBI database showing sequence differences within 3 bp (05-1390, E3191, E2190, FLAC0204, FLAC0181) or cases for which the 16S rRNA sequence could not be identified due to the separation of the contigs (MIN_061107_1834, MIN052511_1280), the sequence of the $16 \mathrm{~S}$ rRNA was the same between strains in this study (Fig. S2). Considering that the average nucleotide identity and alignment percentage of the strains enrolled in this study were greater than $96 \%$ and more than $70 \%$ (Fig. S3), M. paraintracellulare (including $M$. indicus pranii as a strain of $M$. paraintracellulare) and $M$. yongonense should be classified below the rank of the species of $M$. intracellulare rather than a new species.
Consistent to the previous reports [19, 20], M. avium strains were phylogenetically placed much far from the $M$. intracellulare and related strains enrolled in this study (Fig. S4).

\section{Detection of plasmids in clinical strains}

Some $M$. intracellulare strains are known to possess plasmids such as $M$. intracellulare FLAC0181 (plasmid pFLAC0181: NZ_CP023150.1) and $M$. intracellulare subsp. yongonense 05-1390 (pMyong1: NC_020275.1, pMyong2: NC_020276.1). In this study, we found a 24 $\mathrm{kb}$ plasmid in five strains (M002, M006, M008, M023, M025) in the M.i.27 subgroup and a $26 \mathrm{~kb}$ plasmid in the strain M.i.27 (Fig. 2, Fig. S5). These two plasmids were the same, except that the plasmid from M.i.27 (pMi27) possessed a $1.45 \mathrm{~kb}$ insertion sequence in the $24 \mathrm{~kb}$ plasmid possessed by the other five strains. Some of the genes in these plasmids were annotated and included a MerR family transcriptional regulator, an FADdependent pyridine nucleotide-disulfide oxidoreductase, a putative resolvase/invertase/recombinase, and a peptide transporter, but most of the annotated genes were hypothetical. Furthermore, we found that the other 24 kb plasmid in M011 (MP-MIP group) showed high similarity to pFLAC0181 in FLAC0181 (M.i.198 subgroup of TMI group), and a $51 \mathrm{~kb}$ plasmid in M018 (TMI group not belonging to the M.i.198, M.i.27, or ATCC13950 subgroups) showed similarity to the M.i.198 prophage sequence that we reported previously [22]. These data suggested that the plasmids in $M$. intracellulare could be regarded as characterizing subspecies, as well as a tool to analyze the evolution of $M$. intracellulare strains to produce new subspecies by the exchange of plasmids between groups of strains.

\section{Alignment of the genomic regions}

Changes in genomic alignment, such as inversions, are commonly detected among bacteria. Several recent reports revealed genomic inversions in mycobacteria, including $M$. avium $[23,24]$. By contrast, genome alignment was generally preserved among $M$. intracellulare species (Fig. 3, Fig. S6). Inversions were detected in mobile regions such as insertion sequences. The inversion of large sequences was found in MIP MTCC9506 (2709 kb 2968 kb, 3025 kb - 3059 kb, 3114 kb - 3153 kb regions on MTCC9506 genome) as shown previously [16].

\section{Hypermutated regions}

By setting ATCC13950 as the reference strain, several genomic regions were identified as hot-spots of asynonymous hypermutations (Fig. 4, Table 2, Fig. S7, Table S1). Hypermutated regions (300 mutated sites per $10 \mathrm{~kb}$ sequence region) were frequently found, especially among strains in the M.i.27 and MP-MIP groups, some 
Table 1 Genomic features of the M. intracellulare and related strains enrolled in this study

\begin{tabular}{|c|c|c|c|}
\hline & Strain name (GenBank Accession number) & $\begin{array}{l}\text { Best match strain on NCBI references for clinical strains, } \\
\text { or category in NCBI database for NCBI-registered strains }\end{array}$ & $\begin{array}{l}\text { Best match, } \\
\% \text { mapped }\end{array}$ \\
\hline \multirow[t]{31}{*}{ Clinical strains } & M.i.198 & M. intracellulare MOTT-02 & 98 \\
\hline & M.i.27 & M. intracellulare ATCC 13950 & 87 \\
\hline & M001 & M. paraintracellulare & 90 \\
\hline & M002 & M. intracellulare ATCC 13950 & 95 \\
\hline & M003 & M. indicus pranii & 95 \\
\hline & M004 & M. intracellulare MOTT-02 & 98 \\
\hline & M005 & M. intracellulare ATCC 13950 & 94 \\
\hline & M006 & M. intracellulare ATCC 13950 & 92 \\
\hline & M007 & M. intracellulare ATCC 13950 & 94 \\
\hline & M008 & M. intracellulare ATCC 13950 & 89 \\
\hline & M009 & M. intracellulare ATCC 13950 & 90 \\
\hline & M010 & M. intracellulare ATCC 13950 & 96 \\
\hline & M011 & M. indicus pranii & 97 \\
\hline & M012 & M. indicus pranii & 93 \\
\hline & M013 & M. intracellulare ATCC 13950 & 98 \\
\hline & M014 & M. intracellulare ATCC 13950 & 100 \\
\hline & M015 & M. indicus pranii & 95 \\
\hline & M016 & M. intracellulare ATCC 13950 & 95 \\
\hline & M017 & M. paraintracellulare & 92 \\
\hline & M018 & M. intracellulare ATCC 13950 & 85 \\
\hline & M019 & M. paraintracellulare & 92 \\
\hline & M020 & M. paraintracellulare & 92 \\
\hline & M021 & M. paraintracellulare & 92 \\
\hline & M022 & M. intracellulare MOTT-02 & 100 \\
\hline & M023 & M. intracellulare ATCC 13950 & 90 \\
\hline & M024 & M. intracellulare ATCC 13950 & 94 \\
\hline & M025 & M. intracellulare ATCC 13950 & 92 \\
\hline & M026 & M. intracellulare ATCC 13950 & 88 \\
\hline & M027 & M. indicus pranii & 95 \\
\hline & M028 & M. intracellulare ATCC 13950 & 90 \\
\hline & M029 & M. indicus pranii & 93 \\
\hline \multirow[t]{12}{*}{ NCBI-registered strains } & ATCC13950(NC_016946.1) & M. intracellulare & \\
\hline & 1956(JAOG01.1) & M. intracellulare & \\
\hline & CSURP8077(CAAHFM01.1) & M. intracellulare & \\
\hline & FLAC0133(NZ_CP023146.1) & M. intracellulare & \\
\hline & FLAC0181(NZ_CP023149) & M. intracellulare & \\
\hline & 2285(JAOD01.1) & M. intracellulare & \\
\hline & MIN_061107_1834(JAOM01.1) & M. intracellulare & \\
\hline & MIN052511_1280(JAON01.1) & M. intracellulare & \\
\hline & 852002-53206_SCH5915646(LZIO01.1) & M. intracellulare & \\
\hline & E3191(LZJO01.1) & M. intracellulare & \\
\hline & E2190(LZJT01.1) & M. intracellulare & \\
\hline & MOTT-02(NC_016947.1) & M. intracellulare & \\
\hline
\end{tabular}


Table 1 Genomic features of the M. intracellulare and related strains enrolled in this study (Continued)

\begin{tabular}{ll}
\hline Strain name (GenBank Accession number) & $\begin{array}{l}\text { Best match strain on NCBI references for clinical strains, } \\
\text { or category in NCBI database for NCBI-registered strains }\end{array}$ \\
\hline FLAC0204(NSFC01.1) mapped \\
\hline FLAC0162(NSFE01.1)
\end{tabular}

within the same regions and others within different regions. By contrast, hypermutated regions were not found among M.i.198 subgroup strains, with the exception of two consecutive regions in M004 $(670 \mathrm{~kb}-690 \mathrm{~kb}$ regions corresponding to ATCC13950 genome).

The genes of a mammalian cell entry (mce) operon (mce3ABCD-lprM) $[25,26]$ and those involved in fatty acid metabolism ( $a c d 2, f a d E 3, f a d E 22, f a d E 33$ ), a folate synthase $(h m d)$ and those encoding cytochrome $\mathrm{P} 450$ (cyp124, cyp143) were included in the hypermutation regions identified in the clinical strains. The nitroreductase gene $(a c g)$ was commonly found in the hypermutated regions in the strains belonging to the groups M.i.27 and MP-MIP. Genes encoding a transporter (mmpL12) and cytochrome $\mathrm{P} 450$ (cyp123) were found in the hypermutated regions of some strains belonging to the M.i.27 subgroup and the MP-MIP group. Genes involved in DNA and RNA metabolism ( $g y r A, h r p A)$, the phosphatase of trehalose and tyrosine (otsB, lpqD), an immuneregulating protein $(h t d Y)$ [27], a dehydrogenase (zwf), cell wall metabolism (cwsA, mviN), and a type VII secretion system $(e c c C 2)$ were included in the hypermutated regions in the strains of the M.i.27 subgroup. A trehalose glycolipid transporter gene (mmpL13) was found in the hypermutated region in most strains of the MP-MIP group. However, the location of the other hypermutated regions varied from strain to strain. The genes located in hypermutated regions were functionally consistent with the evolution of mycobacteria-specific metabolism, such as the synthesis of mycolic acids and trehalose glycolipids, as well as long-term persistence in the host.

\section{Pan-genomic analysis}

Pan-genomic analysis was performed to calculate the genomic diversity of $M$. intracellulare strains including strains belonging to the TMI, MP-MIP, and $M$. yongonense groups. A prominent feature was that the proportion of accessory genes to core genes (approximately 1900 accessory genes and 3153 core genes) was comparatively higher in the strains enrolled in this study than in $M$. tuberculosis clinical strains (approximately 550 accessory genes and 3679 core genes) (Table S2) [7]. Furthermore, some strains possessed hundreds of unique genes, while others possessed only a few unique genes. Strains possessing a large number of unique genes were dispersed among all subspecies and were not limited to a particular subspecies. When considering the accumulation of new genes in these strains, the exponent of the number of pan-genes expressed by the $\gamma$ parameter from Heaps' law was greater than zero $(0.2990)$ and $M$. intracellulare had 11,513 pan-genes $(n=55)$ indicating an open pan-genome [28] (Fig. 5). At the level of groups of strains, the exponent was decreased but equivalent to that reported for $M$. avium $(\gamma=0.1935)$ indicating an open pan-genome among the groups of strains.

To determine whether the absence of particular genes amongst groups of strains is of value in determining strain-specific virulence factors. In this study, we identified 151, 421, and 766 clusters of exclusively-absent genes in the groups of TMI, MP-MIP, and M. yongonense, respectively, from the total of 290,267 input genes generated by pan-genomic analysis using Bacterial PanGenome Analysis tool (BPGA) software [29]. Of note, with the exception of hypothetical genes, the gene family most commonly associated with exclusively absent genes was the mce gene family (Table 3). Mce functions as a possible ATP-binding cassette transporter in mycobacteria and the number of operons and the levels of sequence similarity among $m c e$ genes are diverse among mycobacteria [25, 26]. In M. intracellulare ATCC13950, the mcel and mce4 operons showed high similarity with those of $m c e 1_{M t b}$ and $m_{c e} 4_{M t b}$ (average $80 \%$ identity) 


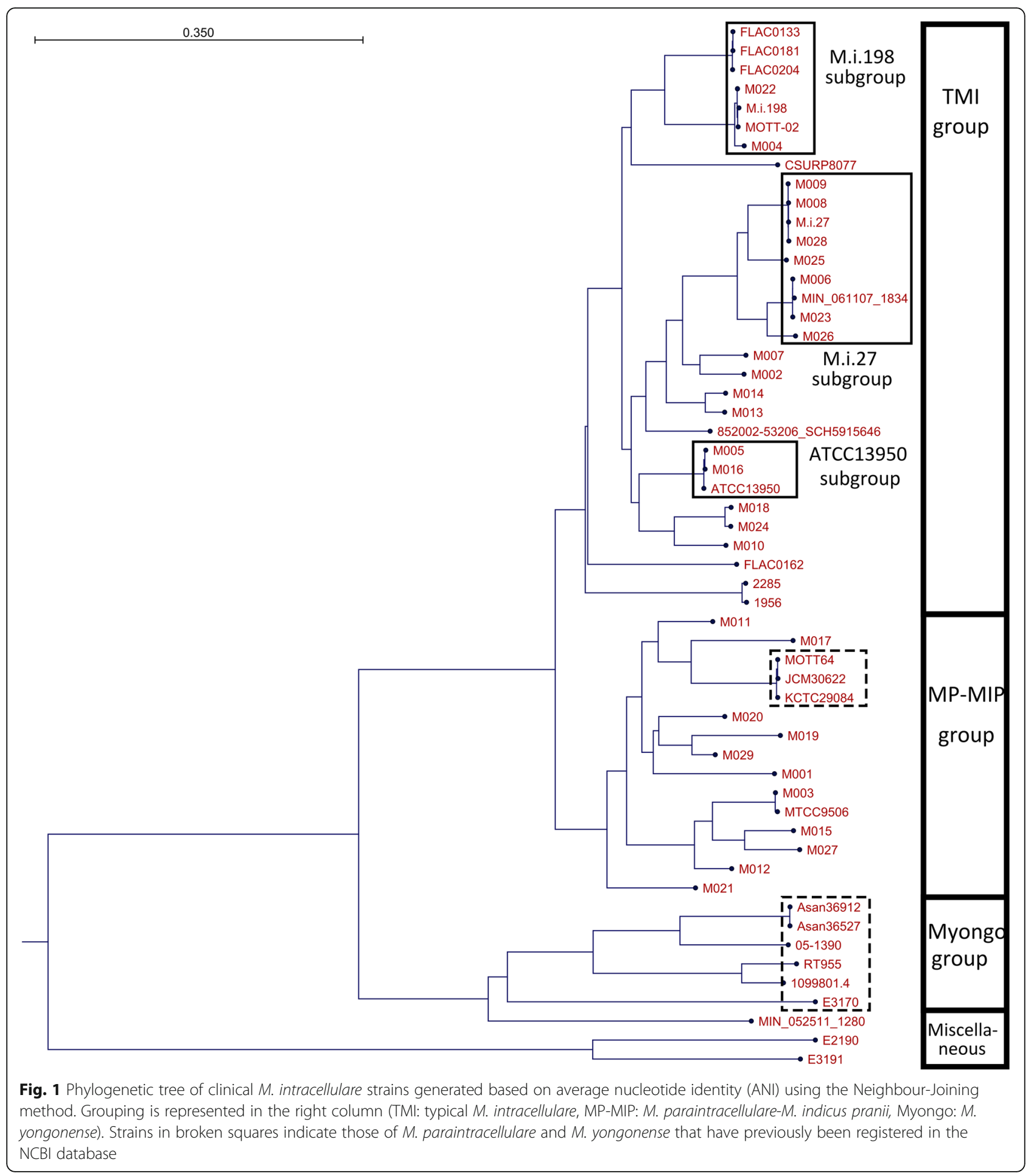

(Table S3). Two mce3 operons existed that showed lower sequence similarity with those of $m c e 3_{M t b}$ (average $65 \%$ identity) and additional three operons existed that also showed low sequence similarity with those of various types of $m c e_{M t b}$ genes $(20-40 \%$ identity). These three operons were phylogenetically grouped into mce7, mce7bis and mce5 of M. smegmatis (Fig. S8). By comparing the sequences of the identified, exclusivelyabsent, mce genes with those of mce sequences in $M$. tuberculosis, M. smegmatis, and M. intracellulare ATCC13950, the composition of mce operons was suggested to be group-specific as follows: i) There was an additional mces operon in the strains of the MP-MIP group and $M$. yongonense not found in those of the TMI 


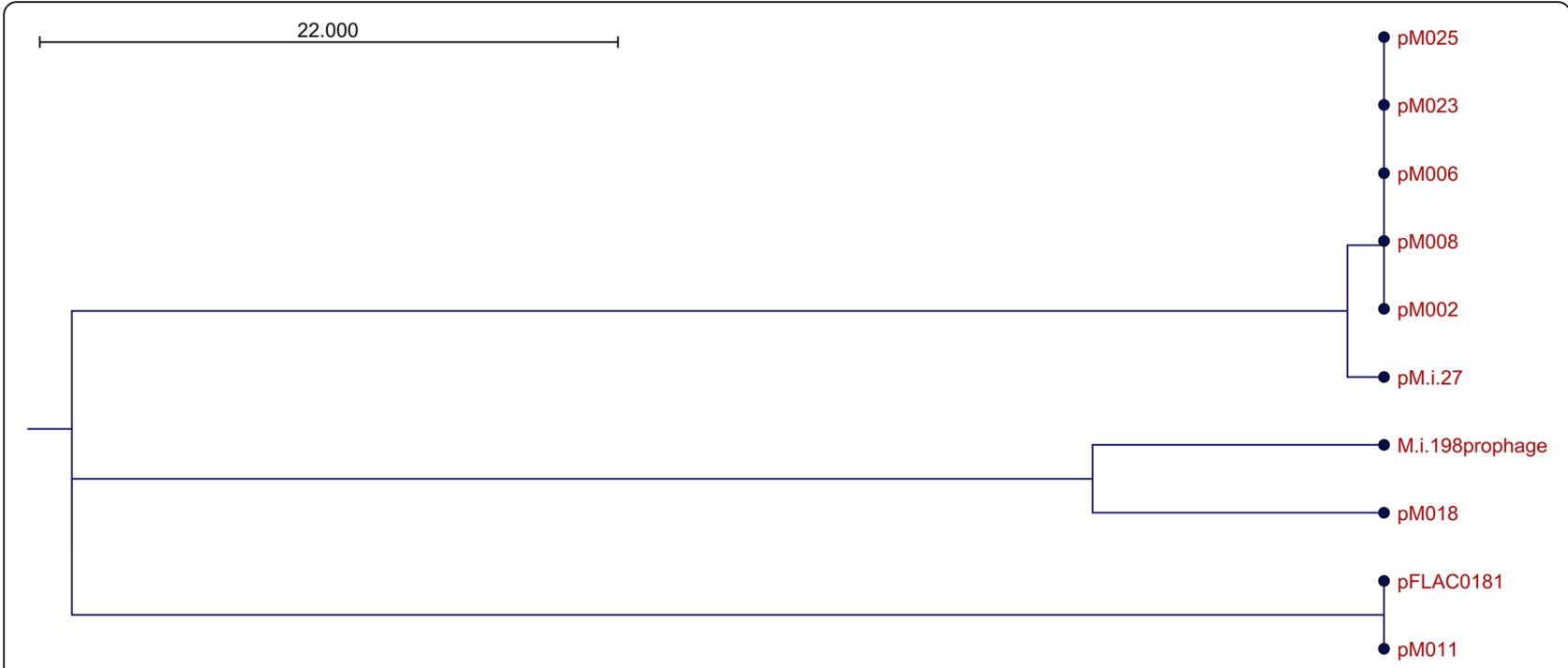

Fig. 2 Phylogenetic tree of the plasmids found in this study. The tree was generated based on alignment percentages (AP) using the Neighbor-Joining method

group. ii) There was an additional mce1 operon in the strains of the TMI group and $M$. yongonense not found in those of the MP-MIP group. iii) The mce3 operons were present only in the strains of the groups of TMI and MP-MIP not found in those of M. yongonense (Table 3, Fig. S9).

\section{Discussion}

In this study, we revealed that clinical $M$. intracellulare strains could be grouped into two distinct groups, namely typical $M$. intracellulare (TMI) and MP-MIP groups, by comparative genomic analysis. Our data were consistent with the previous whole genome sequencing data of a representative strain from each nontuberculous mycobacterial species claiming that $M$. paraintracellulare should be reclassified into $M$. intracellulare at the subspecies level (average nucleotide identity $\geq 98 \%$, alignment percentage $\geq 80 \%$ ) [30-32]. Furthermore, we demonstrated that $M$. indicus pranii MTCC9506 was not classified into the TMI group but into the MP-MIP group. Thus, we propose that the groups of TMI and MP-MIP are reclassified as $M$. intracellulare subsp. intracellulare genovar intracellulare (including the type strain ATCC13950) and genovar paraintracellulare (including the type strain MOTT64), respectively. In this context, we also propose that $M$. indicus pranii MTCC9506 is in fact a strain of $M$. intracellulare subsp. intracellulare genovar paraintracellulare.

Comparative genomic approaches by whole genome sequencing have arisen the reconsideration of the classification of some $M$. intracellulare-related strains. The $M$. yongonense strains and $M$. intracellulare strains enrolled in this study showed synonymous similarity level in the species rank (average nucleotide identity $\geq 96 \%$, alignment percentage $\geq 70 \%$ ), which supports the claim that $M$. yongonense should be reclassified into $M$. intracellulare subsp. yongonense [30-33]. M. chimaera was proposed as a new species of MAC in 2004 from the characteristics of the sequences of 16Sr RNA and 16S23S internal transcribed spacer as well as the unique mycolic acid pattern [34]. M. chimaera seems to have some difference in etiological background from other species causing pulmonary MAC disease because of the reported outbreak in the open-heart surgery patients from the contaminated heater-cooler units [35]. Despite such distinct clinical features, $M$. chimaera has been claimed to be taxonomically synonymous with $M$. intracellulare at the species or subspecies levels (average nucleotide identity $\geq 96 \%$, alignment percentage 79-82\%) $[19,30-32]$. Similar to our study, comparative genomic analyses of a sufficient number of clinical strains may be able to verify the claim.

There are several factors causing taxonomic controversies in $M$. intracaellulare and related strains. The first is the method of defining the bacterial species. Compared to the DNA-DNA hybridization techniques which is still regarded as the gold standard method for analyzing genomic similarities, computational calculation of similarity indices such as average nucleotide identity and alignment percentage provides robust and reproducible data for taxonomic classification. For example, DNA-DNA hybridization value was reported to be $53 \%$ between $M$. paraintracellulare MOTT64 and $M$. intracellulare ATCC13950 in contrast to the computational indices suggesting synonymous subspecies between the two (average nucleotide identity $>98 \%$, predicted DNA-DNA 

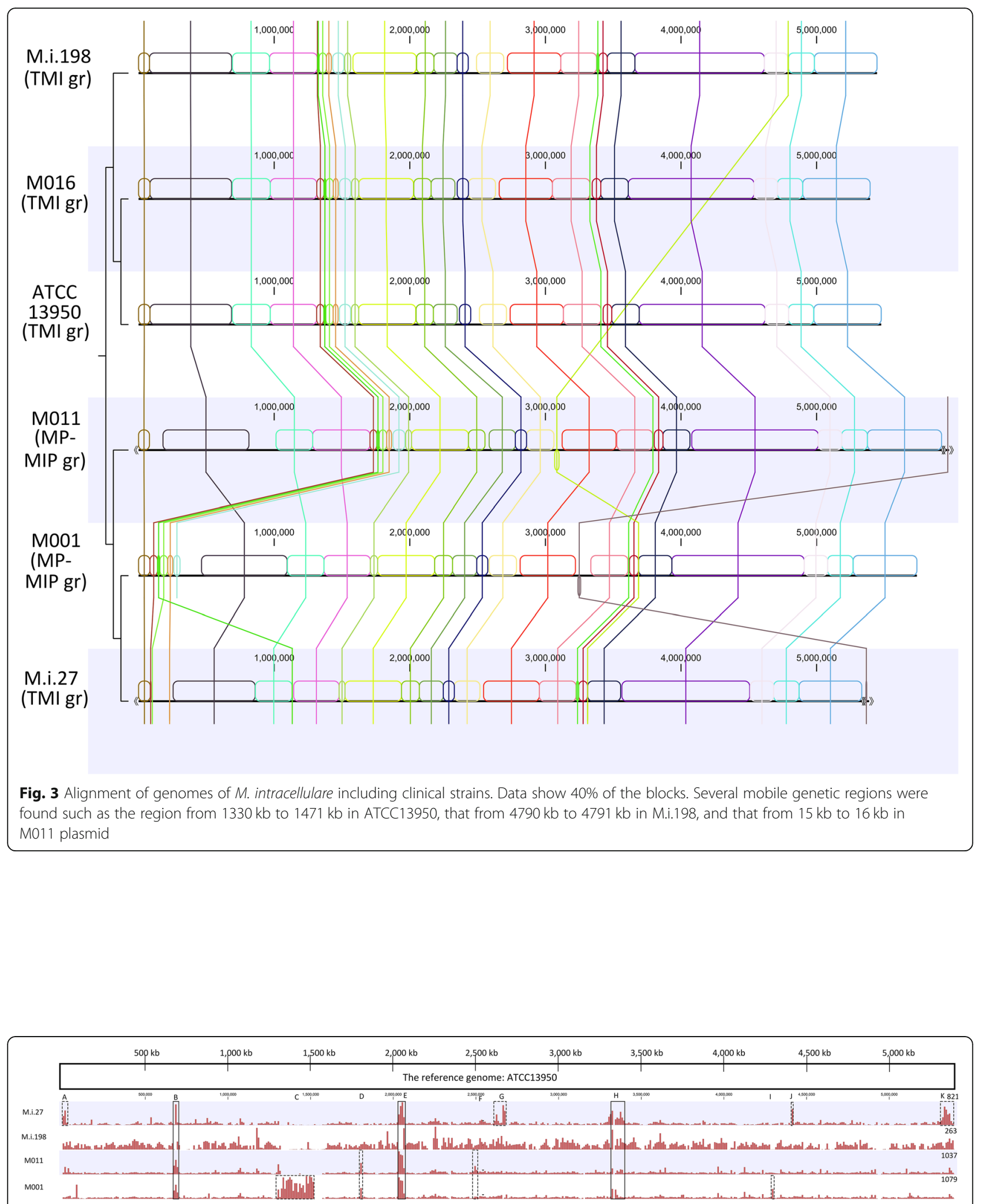

Fig. 4 Distribution of single nucleotide variants (SNV) throughout the genome. The detection of SNV was performed using the genome of ATCC13950 as the reference genome. Numbers on the upper right-hand side for each strain indicate the number of SNV as a scale. Regions surrounded by the bold and dashed lines indicate the common regions $(\mathbf{b}, \mathbf{e}, \mathbf{h})$ and group- or subgroup-specific hypermutated regions $(\mathbf{a}, \mathbf{c}, \mathbf{d}$, $\mathbf{f}, \mathbf{g}, \mathbf{i}, \mathbf{j})$, respectively 
Table $\mathbf{2}$ List of the representative genes located in the hypermutated regions

\begin{tabular}{|c|c|c|}
\hline \multicolumn{2}{|c|}{ M.i.27 subgroup } & \multirow[b]{2}{*}{ Function } \\
\hline Gene & Locus tag $^{*}$ & \\
\hline gyrA & OCU_RS25025 & DNA gyrase subunit $A$ \\
\hline сур 144 & OCU_RS25050 & cytochrome P450 Cyp144 \\
\hline CWSA & OCU_RS25055 & cell wall synthesis protein CwsA \\
\hline citE & OCU_RS37365 & citrate lyase subunit beta \\
\hline$z w f$ & OCU_RS37375 & IMP dehydrogenase \\
\hline hrpA & OCU_RS37390 & ATP-dependent RNA helicase \\
\hline ots $B$ & OCU_RS45365 & trehalose-phosphate phosphatase \\
\hline$h t d Y$ & OCU_RS45370 & 3-hydroxyacyl-thioester dehydratase HtdY \\
\hline IpqD & OCU_RS45375 & histidine phosphatase family protein \\
\hline eccC2 & OCU_RS49810 & type VII secretion protein EccC \\
\hline mviN & OCU_RS49890 & putative peptidoglycan biosynthesis protein MviN \\
\hline$r s m A$ & OCU_RS49900 & anti-sigma-M factor RsmA \\
\hline trxB_2 & OCU_RS49905 & thioredoxin reductase \\
\hline \multicolumn{3}{|c|}{ MP-MIP group } \\
\hline Gene & Locus tag ${ }^{*}$ & Function \\
\hline paaG & OCU_RS31460 & enoyl-CoA hydratase \\
\hline mce3A & OCU_RS31625 & MCE-family protein Mce $3 \mathrm{~A}$ \\
\hline mсезB & OCU_RS31630 & Mce family protein Mce3B \\
\hline mсезC & OCU_RS31635 & Mce family protein Mce3C \\
\hline mce3D & OCU_RS31640 & Mce family protein Mce3D \\
\hline IprM & OCU_RS31645 & MCE-family protein Mce3E \\
\hline hmd & OCU_RS31690 & 5,10-methylene tetrahydromethanopterin reductase \\
\hline cyp 143 & OCU_RS31715 & putative cytochrome P450 Cyp143 \\
\hline fadE3 & OCU_RS31935 & acyl-CoA dehydrogenase \\
\hline acd & OCU_RS32010 & acyl-CoA dehydrogenase \\
\hline fadE33 & OCU_RS32080 & acyl-CoA dehydrogenase \\
\hline cyp 124 & OCU_RS32180 & methyl-branched lipid omega-hydroxylase \\
\hline$m h p B$ & OCU_RS32235 & 2,3-dihydroxyphenylpropionate/2,3-dihydroxicinnamic acid 1,2-dioxygenase \\
\hline cyp 143 & OCU_RS32255 & putative cytochrome P450 Cyp143 \\
\hline fadE22 & OCU_RS32275 & acyl-CoA dehydrogenase FadE22 \\
\hline mmpL13 & OCU_RS33545 & Transport protein MmpL13 \\
\hline \multicolumn{3}{|c|}{ Both M.i.27 subgroup and MP-MIP group } \\
\hline Gene & Locus tag ${ }^{*}$ & Function \\
\hline mmpL12 & OCU_RS28435 & putative transport protein MmpL12 \\
\hline cyp123 & OCU_RS28470 & putative cytochrome P450 Cyp123 \\
\hline $\operatorname{acg}$ & OCU_RS34620 & putative NADPH nitroreductase Acg \\
\hline
\end{tabular}

"Locus tag assigned on ATCC13950

hybridization value calculated from genome blast distance phylogeny $>80 \%$ ) [13]. And the genomic similarity has been verified by the following whole genome sequencing data including ours $[19,31,32]$. The second is which strain is chosen as a representative strain from each species for comparative genomic analyses. Our data were consistent with the study by Tortoli showing that
M. intracellulare and M. paraintracellulare are monophyletic and $M$. yongonense is paraphyletic to $M$. intracellulare and M. paraintracellulare [19]. On the other hand, our data were different from the study by Matsumoto showing that $M$. indicus pranii and $M$. intracellulare are monophyletic but $M$. intracellulare and $M$. paraintracellulare are paraphyletic [20]. The former 


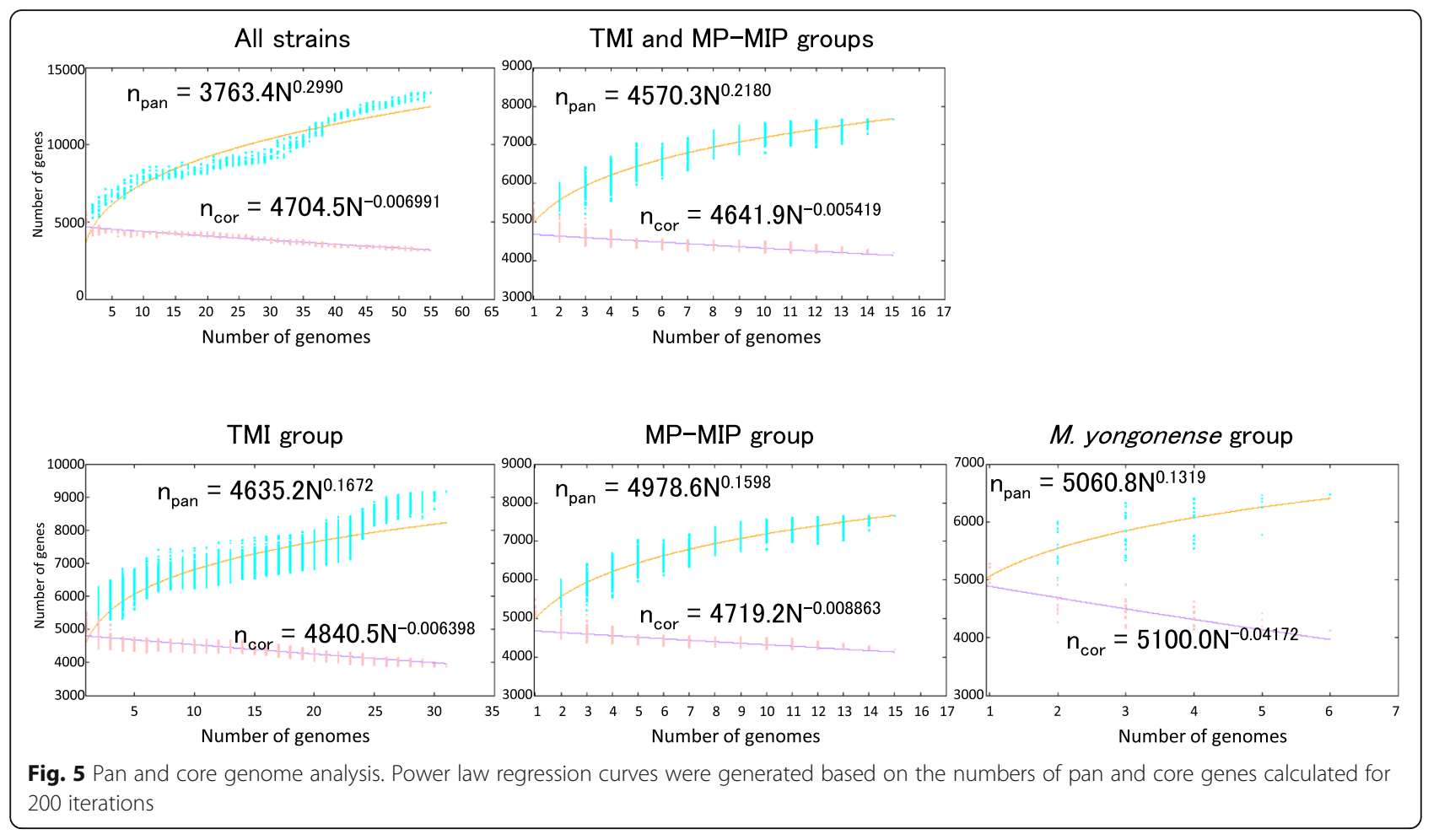

study chose $M$. intracellulare ATCC13950, a type strain of TMI, but the latter study chose $M$. intracellulare MIN_052511_1280, a miscellaneous strain neither belonging to the TMI group nor the MP-MIP group. The different choice of the strains enrolled for analyses causes taxonomic discrepancies. Comparative genomic analysis enrolling a sufficient number of clinical strains is considered to be an important step in taxonomic studies. The third is the relationship between genomic similarities and other clinical features including phenotypes and epidemiology. Based on the genomic similarity data, some species within the $M$. tuberculosis complex (i.e. $M$. africanum, M. bovis, M. caprae, M. microti and M. pinnipedii) have been reclassified as $M$. tuberculosis [36]. Similar to the case of $M$. tuberculosis, M. yonogonense and $M$. chimaera have been proposed to be reclassified as synonymous with each other at the species or subspecies levels [19, 30-32]. Such reclassification may increase the species and subspecies categories that include mycobacteria with distinct clinical features. How to integrate the information of clinical features into the phylogenetic data based on genomic similarities should be further investigated for consistent taxonomic classification and nomenclature between the fields of bacteriology and clinical infectious disease.

Previous studies using multilocus sequence typing of housekeeping genes demonstrated the obscure classification of TMI strains with distinct sub-grouping between MP and MIP [18, 37]. We found that the subgroups of
M.i.198 (including MOTT-02) and ATCC13950 were barely classified by multilocus sequence typing but these were phylogenetically very close (Fig. S10). The strains of the MP-MIP subgroup were distinctly classified into the two groups (one included $M$. paraintracellulare MOTT64 and the other included $M$. indicus pranii MTCC9506). However, the branching pattern of each strain was different from the genomic phylogeny. The strains of the M.i.27 subgroup were not able to be classified by the sequence-based genotyping because they were placed on the root of the phylogenic trees. Taken together, whole genome sequencing method should be recommended for modernizing clinical taxonomy.

Several types of plasmids were detected among clinical M. intracellulare strains. Plasmid pMI27 and its related plasmids found in the M.i.27 subgroup were novel. However, pMI27 was not detected in every strain belonging to the M.i.27 subgroup, suggesting heterogeneity in their plasmid profiles. The identification of similar plasmids among the different groups and subgroups of strains, such as pFLAC0181 in M011 and M.i.198 prophage in M018, seems to infer the possible transmission of the sequence during strain evolution.

Mycobacteria are characterized by their unique lipid metabolism involving the synthesis of long-chain fatty acids such as mycolic acids. The inclusion of several genes of fatty acid metabolism ( $f a d E 3, f a d E 33)$ in the hypermutation hot-spot regions suggested the adaptation to survive in the host. Similarly, the inclusion of 
Table 3 Group specificity of the mce genes in terms of the detection of the exclusively absent clusters of genes by pan-genomic analysis

\begin{tabular}{|c|c|c|}
\hline \multicolumn{3}{|l|}{ Absent from TMI group } \\
\hline Derived strain (strain group) of hit cluster & Possible function & Homologous genes in ATCC13950 \\
\hline MOTT64(MP-MIP) & virulence factor Mce & OCU_RS29085 \\
\hline MOTT64(MP-MIP) & mammalian cell entry protein & OCU_RS29065 \\
\hline KCTC29084(MP-MIP) & mammalian cell entry protein & OCU_RS29070 \\
\hline JCM30622(MP-MIP) & mammalian cell entry protein & OCU_RS29075 \\
\hline JCM30622(MP-MIP) & virulence factor Mce family protein & OCU_RS29060 \\
\hline 1099801.4(M. yongonense) & virulence factor Mce & OCU_RS29080 \\
\hline \multicolumn{3}{|l|}{ Absent from MP-MIP group } \\
\hline Derived strain (strain group) of hit cluster & Possible function & Homologous genes in ATCC13950 \\
\hline M023(TMI) & mammalian cell entry protein & OCU_RS48855 \\
\hline FLAC0162(TMI) & Mce family protein Mce1B & OCU_RS48875 \\
\hline 1099801.4(M. yonogonense) & Mce family protein Mce1C & OCU_RS48870 \\
\hline 05-1390(M. yonogonense) & Mce family protein Mce2A & OCU_RS28225 \\
\hline 05-1390(M. yonogonense) & Mce family protein Mce1D & OCU_RS48865 \\
\hline \multicolumn{3}{|l|}{ Absent from $M$. yongonense group } \\
\hline Derived strain (strain group) of hit cluster & Possible function & Homologous genes in ATCC13950 \\
\hline M021(MP-MIP) & mammalian cell entry protein & OCU_RS31630 \\
\hline M.i.27(TMI) & Mce family protein Mce3B & OCU_RS32530 \\
\hline M023(TMI) & mammalian cell entry protein & OCU_RS32545 \\
\hline M025(TMI) & Mce family protein Mce3D & OCU_RS31640 \\
\hline M025(TMI) & Mce associated membrane protein & OCU_RS32555 \\
\hline M026(TMI) & mammalian cell entry protein & OCU_RS31650 \\
\hline M028(TMI) & Mce family protein Mce3D & OCU_RS32540 \\
\hline M010(TMI) & Mce family protein Mce3C & OCU_RS32535 \\
\hline M017(MP-MIP) & Mce family protein Mce1D & OCU_RS48865 \\
\hline M017(MP-MIP) & mammalian cell entry protein & OCU_RS48860 \\
\hline M017(MP-MIP) & Mce associated protein & OCU_RS48840 \\
\hline M012(MP-MIP) & mammalian cell entry protein & OCU_RS32550 \\
\hline CSURP8077(TMI) & Mce family protein Mce1B & OCU_RS48875 \\
\hline CSURP8077(TMI) & Mce family protein Mce1C & OCU_RS48870 \\
\hline CSURP8077(TMI) & mammalian cell entry protein & OCU_RS48855 \\
\hline 2285(TMl) & Mce family protein Mce3C & OCU_RS31635 \\
\hline 1956(TMI) & MCE-family protein MCE3A & OCU_RS31625 \\
\hline FLAC0181(TMI) & Mce associated membrane protein & OCU_RS31655 \\
\hline
\end{tabular}

genes encoding lipid transporters (eccB2, eccC2, $m c e 3$ operon) in the hypermutation hot-spot regions in the strains of the M.i.27 subgroup and the strains of the MP-MIP group suggested adaptation in the uptake of lipids to ensure survival in the host [38].

By including the data for clinical strains, M. intracellulare was proven to be pan-genomic, similar to other mycobacteria, such as M. avium and M. tuberculosis [6, $7,9]$. The comparatively higher proportion of non-core genes to core genes in $M$. intracellulare suggested higher genomic evolutionary activity than in M. tuberculosis, which may be associated with the diversity of bacteriological characteristics (colony appearance, growth rate in medium, and experimental virulence) and clinical manifestations [21, 39-41]. In this study, the mce operons were found to be a group-specific gene family. Mce proteins constitute an $\mathrm{ABC}$ transporter in mycobacteria thought to transport phospholipids, based on homology to the Mce4 system in M. tuberculosis that transports cholesterol and is required for persistent in vivo 
infection [42]. The mce1 operon has been suggested to be a mycolic acid re-importer [43] but the function of the other mce operons has not been elucidated. Identifying the mce operons as group-specific genes is an important step in elucidating the virulence factors in $M$. intracellulare and its related strains. In particular, the mce1 operon seems to be a promising candidate of virulence factors with evidence of its role in hypoxic biofilm formation, as revealed by the genome-wide identification of essential genes by transposon sequencing [44].

In $M$. yongonense, the lateral gene transfer event has been suggested in $r р о B$ gene from a distantly related scotochromogen $M$. parascrofulaceum by multi-locus sequence typing [45]. Taking this notion into consideration, the accumulation of lateral gene transfer events may explain the incongruence of the phylogenetic trees based on limited housekeeping genes and those based on genomic sequences. On the other hand, in genomic level, the impact of lateral gene transfer on genomic evolution has been estimated to be low in mycobacteria because the percentage of genomes resulting from lateral gene transfer is low (ranging from 0.04 to $1.9 \%$ ) without large recombination (>1 kb) [46]. To overcome the complexity of the taxonomic classification by the lateral gene transfer, the prior use of whole genomic sequencing data should be recommended for discussing genomic features of clinical strains, rather than the simple use of genotyping data of housekeeping genes.

Our data provide basic information on the genetic similarity and diversity of clinical $M$. intracellulare strains that enables a better understanding of the evolution of strains causing pulmonary MAC disease. However, this study has some limitations. The population size of this study was small and there were some strains that belonged outside of the TMI or MP-MIP groups (E2190, E3191) and did not belong to the major subgroups of M.i.27, ATCC13950, and M.i.198. Characterization of these orphan strains may be of value to fully elucidate the relationship between genomic differences and pathogenic phenotypes in pulmonary MAC disease. The typing of genome sequences of a collection of Japanese clinical isolates is ongoing. The addition of newly isolated strains from $M$. intracellulare endemic areas, such as the US and India, may also give some insight into the genomic characteristics of this pathogen globally $[2,4]$. The biological significance of the groupspecific genes identified by comparative genomics remains to be determined. In contrast to M. tuberculosis, biological experiments using deletion mutants have not been reported for pathogenic mycobacteria, with the exception of M. marinum that mimics M. tuberculosis as it possesses the ESX-1 secretion system [47]. We have recently modified a gene manipulation method for $M$. intracellulare using mycobacterial plasmids and phages developed for M. tuberculosis [44]. The next step will be to analyze the biological effects in these deletion mutants to fully elucidate the molecular mechanisms of pulmonary MAC disease.

\section{Conclusions}

We have clarified the major groups of $M$. intracellulare by comparative genomic analysis of the clinical strains derived from pulmonary MAC disease patients and previously-reported genome sequences. Furthermore, we have clarified the diversity of $M$. intracellulare by pangenomic analysis and the mce operons were identified as contributing to the genomic diversity. These data provide vital genetic information that can be used to elucidate the virulence factors and mechanisms of pathogenesis in recently-emerged non-tuberculous mycobacterial disease.

\section{Methods \\ Study subjects}

A total of 29 non-HIV patients with pulmonary MAC disease were enrolled in this study between January 2015 and March 2019. All patients were either treated as outpatients or were hospitalized in the National Hospital Organization Osaka Toneyama Medical Center. This study was approved by the Institutional Review Board of the National Hospital Organization Osaka Toneyama Medical Center and Niigata University Hospital. The opportunity to opt-out of consent was provided for all participants. The diagnosis of MAC disease was conducted based on the diagnostic guidelines proposed by the American Thoracic Society $[39,40]$. The diagnosis of clinical specimens was performed by a DNA-DNA hybridization assay using AccuProbe (Gen-Probe Inc., San Diego, CA) or COBAS AMPLICOR (Roche Diagnostic, Tokyo, Japan) systems or by DNA-DNA hybridization assay (Kyokuto Pharmaceutical Industrial, Tokyo, Japan). After treatment with $\mathrm{NaOH}$ and $\mathrm{N}$ acetyl-L-cysteine, the sputum samples were streaked onto Lowenstein-Jensen medium. Single colonies were isolated on 7H10/OADC agar plates. One isolated strain per patient was analyzed in this study. Two additional clinical M. intracellulare strains M.i.198 and M.i.27, which had previously been reported to be virulent in mice, were included in this study [21]. Genome sequence data for strains registered by other researchers in the NCBI database were obtained and used in this study.

\section{Genome sequencing}

Genomic DNA from the clinical strains was isolated by the phenol-chloroform method as previously described [48]. Sequencing was performed using the MiSeq system and PacBio RS-II. Sequencing libraries for MiSeq were prepared using the Nextera XT DNA Library 
Preparation Kit according to the manufacturer's protocol (Illumina, CA). Each DNA library with adapters was normalized to $4 \mathrm{nM}$, pooled, and sequenced by the MiSeq system with MiSeq Reagent Kit v3 (Illumina, CA). Sequencing libraries for PacBio were prepared using SMRTbell Template Prep Kit 1.0 according to the manufacturer's protocol (Pacific Biosciences, CA). For each SMRTbell DNA library, size selection was performed using BluePippin. Each size selected SMRTbell DNA library was sequenced by the PacBio RS-II system with DNA Sequencing Reagent Kit 4.0 v 2 (Pacific Biosciences, CA). All genome sequences, including the strains registered by other researchers in $\mathrm{NCBI}$, were annotated using dFAST to standardize the data prior to pangenomic analysis [49].

\section{Analysis of genome sequence data}

Genome sequence data were handled by the CLC Genomics Workbench system (Qiagen Inc., Valencia, CA). Species identification was performed based on the genome data by finding the best match species from the NCBI bacteria database (downloaded on June 25, 2020). A complete genome sequence was obtained by mapping the reads obtained by MiSeq to those obtained by PacBio and the consensus sequence was adopted as the complete genome sequence if the sequence could be finished as a circular chromosome with/without a circular plasmid. M. intracellulare ATCC13950 was set as the reference strain. The whole genome sequences were aligned using the analytic tool provided by the CLC Genomics Workbench system, with the following default settings: minimum initial seed length 15 , allow mismatches in seeds, minimum alignment block length 100 . The identification of asynonymous single nucleotide polymorphisms was performed using the analytic tools provided by the same Workbench system.

Pan-genomic analysis, including the identification of exclusively-absent genes in the designated groups of strains, was performed using Bacterial Pan-Genome Analysis tool (BPGA) software [29]. Clustering of the genes was performed using USEARCH on BPGA with cut-off sequence identity set at $80 \%$. Pan-genome profile calculations were performed with 200 iterations of combinations.

\section{Supplementary Information}

The online version contains supplementary material available at https://doi. org/10.1186/s12866-021-02163-9.

\section{Additional file 1.}

Additional file 2.

Additional file 3.

Additional file 4.

\section{Acknowledgements}

The authors thank for Ms. Haruko Saito and Dr. Takanori Matsuki for preparing clinical specimens.

\section{Authors' contributions}

Y.T. conceived, designed the experiments. M.M. and R.M. contributed samples. Y.T., Y.F. and C.N. performed the experiments. Y.T., Y.F., C.N., Y.S. and S.M. analyzed the data. Y.T., Y.O., A.N., Y.F., C.N., Y.S. and S.M. contributed reagents/analysis tools. Y.T., Y.F. C.N., Y.S. and S.M. wrote the paper. All authors read and approved the final manuscript.

\section{Funding}

This work was supported by Grant-in-Aid for Scientific Research (grant number 18 K0812 to Yoshitaka Tateishi) from Ministry of Health, Labour and Welfare and the Research Program on Emerging and Re-emerging Infectious Disease from the Japan Agency for Medical Research and Development, AMED (grant number J20J0058 to Sohkichi Matsumoto, JP19fk0108043h0703 to Mari Miki). The research was also supported in part by a grant from the Ministry of Education, Culture, Sports, Science and Technology, Japan (MEXT) for the Joint Research Program of the Research Center for Zoonosis Control, Hokkaido University, and in part by Japan Agency for Medical Research and Development (AMED) (grant number JP20jk0210005 to Sohkichi Matsumoto and Yasuhiko Suzuki).

\section{Availability of data and materials}

The datasets generated in this study are deposited in the DNA Data Bank of Japan (DDBJ) (https://ddbj.nig.ac.jp/), Accession Number: DRA011116. The data deposited is publicly available.

\section{Declarations}

\section{Ethics approval and consent to participate}

This study was approved by the Institutional Review Board of the National Hospital Organization Osaka Toneyama Medical Center (TNH-2019006) and Niigata University Hospital (2019-0020). This study was conducted in accordance with the Declaration of Helsinki. Informed consent was obtained from all participants.

\section{Consent for publication}

Not applicable.

\section{Competing interests}

The authors declare that they have no competing interests.

\section{Author details}

'Department of Bacteriology, Graduate School of Medical and Dental Sciences, Niigata University, Niigata, Japan. ${ }^{2}$ Department of Respiratory Medicine, National Hospital Organization Osaka Toneyama Medical Center, Toyonaka, Osaka, Japan. ${ }^{3}$ Graduate School of Health Care Sciences, Jikei Institute, Osaka, Japan. ${ }^{4}$ Division of Bioresources, Hokkaido University Research Center for Zoonosis Control, Sapporo, Japan. ${ }^{5}$ International Collaboration Unit, Research Center for Zoonosis Control, Hokkaido University, Sapporo, Japan. ${ }^{6}$ Laboratory of Tuberculosis, Institute of Tropical Disease, Universitas Airlangga, Kampus C Jl. Mulyorejo, Surabaya, Indonesia.

Received: 19 November 2020 Accepted: 18 March 2021

Published online: 06 April 2021

References

1. Brode SK, Caley CL, Marras TK. The epidemiologic relationship between tuberculosis and non-tuberculous mycobacterial disease: a systematic review. Int J Tuberc Lung Dis. 2014;18(11):1370-7. https://doi.org/10.5588/ ijtld.14.0120

2. Adjemian J, Olivier KN, Seitz AE, Holland SM, Prevots DR. Prevalence of nontuberculous mycobacterial lung disease in U.S. Medicare beneficiaries. Am J Respir Crit Care Med. 2012;185(8):881-6. https://doi.org/10.1164/rccm.2 01111-20160C.

3. Raju R, Raju SM, Zhao Y, Rubin EJ. Leveraging advances in tuberculosis diagnosis and treatment to address nontuberculous mycobacterial disease. Emerg Infect Dis. 2016;22(3):365-9. https://doi.org/10.3201/eid2203.151643. 
4. Cowman S, van Ingen J, Griffith DE, Loebinger MR. Non-tuberculous mycobacterial pulmonary disease. Eur Respir J. 2019;54(1):1900250. https:// doi.org/10.1183/13993003.00250-2019.

5. Namkoong H, Kurashima A, Morimoto K, Hoshino $Y$, Hasegawa N, Ato M, et al. Epidemiology of pulmonary nontuberculous mycobacterial disease, Japan. Emerg Infect Dis. 2016;22(6):1116-7. https://doi.org/10.3201/eid2206.1 51086.

6. Periwal Y, Patowary A, Vellarikkal SK, Gupta A, Singh M, Mittal A, et al. Comparative whole-genome analysis of clinical isolates reveals characteristic architecture of Mycobacterium tuberculosis pangenome. PLoS One. 2015; 10(4):e0122979. https://doi.org/10.1371/journal.pone.0122979.

7. Yang T, Zhong J, Zhang J, Li C, Yu X, Xiao J, et al. Pan-genomic study of Mycobacterium tuberculosis reflecting the primary/secondary genes, generality/ individuality, and the interconversion through copy number variations. Front Microbiol. 2018;9:1886. https://doi.org/10.3389/fmicb.2018.01886.

8. Das S, Pettersson BMF, Behra PRK, Mallick A, Cheramie M, Ramesh M, et al. Extensive genomic diversity among Mycobacterium marinum strains revealed by whole genome sequencing. Sci Rep. 2020;10:5246.

9. Uchiya K, Tomida S, Nakagawa T, Asahi S, Nikai T, Ogawa K. Comparative genome analyses of Mycobacterium avium reveal genomic features of its subspecies and strains that cause progression of pulmonary disease. Sci Rep. 2017;7(1):39750. https://doi.org/10.1038/srep39750.

10. Rindi L, Garzelli C. Genetic diversity and phylogeny of Mycobacterium avium. Infect Genet Evol. 2014;21:375-83. https://doi.org/10.1016/j.meegid.2013.12.007.

11. Mijs W, de Haas P, Rossau R, Van der Laan T, Rigouts L, Portaels F, et al. Molecular evidence to support a proposal to reserve the designation Mycobacterium avium subsp avium for bird-type isolates and 'M. avium subsp. hominissuis' for the human/porcine type of M. avium. Int. J. Syst. Evol. Microbiol. 2002:52:1505-18.

12. Thorel MF, Krichevsky M, Lévy-Frébault W. Numerical taxonomy of mycobactin-dependent mycobacteria, emended description of Mycobacterium avium, and description of Mycobacterium avium subsp. avium subsp. nov., Mycobacterium avium subsp. paratuberculosis subsp. nov., and Mycobacterium avium subsp. silvaticum subsp. nov. Int J Syst Bacteriol. 1990;40(3):254-60. https://doi.org/10.1099/00207713-40-3-254.

13. Lee S-Y, Kim B-J, Kim H, Won Y-S, Jeon CO, Jeong J, et al. Mycobacterium paraintracellulare sp. nov., for the genotype INT-1 of Mycobacterium intracellulare. Int J Syst Evol Microbiol. 2016;66(8):3132-41. https://doi.org/1 0.1099/ijsem.0.001158.

14. Kim B-J, Math RK, Jeon CO, Yu H-K, Park Y-G, Kook Y-H, et al. Mycobacterium yongonense sp. nov., a slow-growing non-chromogenic species closely related to Mycobacterium intracellulare. Int J Syst Evol Microbiol. 2013;63(Pt_ 1):192-9. https://doi.org/10.1099/ijs.0.037465-0.

15. Saini V, Raghuvanshi S, Talwar GP, Ahmed N, Khurana JP, Hasnain SE, et al. Polyphasic taxonomic analysis establishes Mycobacterium indicus pranii as a distinct species. PLoS One. 2009;4(7):e6263. https://doi.org/10.1371/journal. pone.0006263.

16. Saini V, Raghuvanshi S, Khurana JP, Ahmed N, Hasnain SE, Tyagi AK, et al. Massive gene acquisitions in Mycobacterium indicus pranii provide a perspective on mycobacterial evolution. Nucleic Acids Res. 2012;40(21): 10832-50. https://doi.org/10.1093/nar/gks793.

17. Kim B-J, Choi B-S, Lim J-S, Choi I-Y, Kook Y-H, Kim B-J. Complete genome sequence of Mycobacterium intracellulare clinical strain MOTT-64, belonging to the INT1 genotype. J Bacteriol. 2012;194(12):3268. https://doi.org/10.1128/ JB.00471-12.

18. Kim S-Y, Park HY, Jeong B-H, Jeon K, Huh HJ, Ki C-S, et al. Molecular analysis of clinical isolates previously diagnosed as Mycobacterium intracellulare reveals incidental findings of "Mycobacterium indicus pranii" genotypes in human ling infection. BMC Infect Dis. 2015;15(1):406. https://doi.org/10.11 86/s12879-015-1140-4.

19. Tortoli E, Fedrizzi T, Meehan CJ, Trovato A, Grottola A, Giacobazzi R, et al. The new phylogeny of the genus Mycobacterium: the old and the news. Infect Genet Evol. 2017;56:19-25. https://doi.org/10.1016/j.meegid.2017.10.013.

20. Matsumoto Y, Kinjo T, Motooka D, Nabeya D, Jung N, Uechi K, et al. Comprehensive subspecies identification of 175 nontuberculous mycobacteria species based on 7547 genomic profiles. Emerg Microbes Infect. 2019;8(1):1043-53. https://doi.org/10.1080/22221751.2019.1637702.

21. Tateishi $Y$, Hirayama $Y$, Ozeki $Y$, Nishiuchi $Y$, Yoshimura M, Kang J, et al. Virulence of Mycobacterium avium complex strains isolated from immunocompetent patients. Microb Pathog. 2009;46(1):6-12. https://doi. org/10.1016/j.micpath.2008.10.007.
22. Tateishi Y, Kitada S, Miki K, Maekura R, Ogura Y, Ozeki Y, et al. Wholegenome sequence of the hypervirulent clinical strain Mycobacterium intracellulare M.i.198. J Bacteriol. 2012;194:6336.

23. Uchiya $\mathrm{K}$, Takahashi $\mathrm{H}$, Yagi $\mathrm{T}$, Moriyama M, Inagaki $\mathrm{T}$, Ishikawa $\mathrm{K}$, et al. Comparative genome analysis of Mycobacterium avium revealed genetic diversity in strains that cause pulmonary and disseminated disease. PLoS One. 2013;8(8):e71831. https://doi.org/10.1371/journal.pone.0071831.

24. Bannantine JP, Wu CW, Hsu C, Zhou S, Schwartz DC, Bayles DO, et al. Genome sequencing of ovine isolates of Mycobacterium avium subspecies paratuberculosis offers insights into host association. BMC Genomics. 2012; 13(1):89. https://doi.org/10.1186/1471-2164-13-89.

25. Casali N, Riley LW. A phylogenomic analysis of the Actinomycetales mce operons. BMC Genomics. 2007;8(1):60. https://doi.org/10.1186/1471-2164-8-60.

26. Hemati Z, Derakhshandeh A, Haghkhah M, Chaubey KK, Gupta S, Singh M, et al. Mammalian cell entry operons; novel and major subset candidates for diagnosis with special reference to Mycobacterium avium subspecies paratuberculosis infection. Vet Quartery. 2019;39(1):65-75. https://doi.org/1 0.1080/01652176.2019.1641764.

27. Zao J-W, Sim Z-Q, Zhang X-Y, Zhang Y, Liu J, Ye J, et al. Mycobacterial 3hydroxyacyl-I-thioester dehydratase $Y$ derived from Mycobacterium tuberculosis induces COX-2 expression in mouse macrophages through MAPK-NF-KB pathway. Immunol Lett. 2014;161(1):125-32. https://doi.org/1 0.1016/j.imlet.2014.05.013.

28. Tettelin H, Riley D, Cattuto C, Medini D. Comparative genomics: the bacterial pan-genome. Curr Opin Microbiol. 2008;11(5):472-7. https://doi. org/10.1016/j.mib.2008.09.006.

29. Chaudhari NM, Gupta VK, Dutta C. BPGA- an ultra-fast pan-genome analysis pipeline. Sci Rep. 2016;6(1):24373. https://doi.org/10.1038/srep24373.

30. van Ingen J, Turenne CY, Tortoli E, Wallace RJ Jr, Brown-Elliott BA. A definition of the Mycobacterium avium complex for taxonomical and clinical purposes, a review. Int J Syst Evol Microbiol. 2018;68(11):3666-77. https:// doi.org/10.1099/ijsem.0.003026.

31. Nouioui I, Carro L, García-López M, Meier-Kolthoff JP, Woyke T, Kyrpides NC, et al. Genome-based taxonomic classification of the phylum Actinobacteria. Front Microbiol. 2018;9:2007. https://doi.org/10.3389/fmicb.2018.02007.

32. Tortoli E, Meehan CJ, Grottola A, Fregni Serpini G, Fabio A, Trovato A, et al. Genome-based taxonomic revision detects a number of synonymous taxa in the genus Mycobacterium. Infect Genet Evol. 2019;75:103983. https://doi. org/10.1016/j.meegid.2019.103983.

33. Castejon M, Menéndez MC, Comas I, Vicente A, Garcia MJ. Whole-genome sequence analysis of the Mycobacterium avium complex and proposal of the transfer of Mycobacterium yongonense to Mycobaterium intracellulare subsp. yonogense susbp. Int J System Evol Microbiol. 2018;68(6):1998-2005. https://doi.org/10.1099/ijsem.0.002767.

34. Tortoli E, Rindi L, Garcia MJ, Chiaradonna P, Dei R, Garzelli C, et al. Proposal to elevate the genetic variant MAC-A, included in the Mycobacterium avium complex, to species rank as Mycobacterium chimaera sp. nov. Int J Syst Evol Microbiol. 2004;54(4):1277-85. https://doi.org/10.1099/ijs.0.02777-0.

35. Kasperbauer SH, Daley CL. Mycobacterium chimaera infections related to the heater-cooler unit outbreak: A guide to diagnosis and management. Clin Infect Dis. 2019;68(7):1244-50. https://doi.org/10.1093/cid/ciy789.

36. Riojas MA, McGough KJ, Rider-Riojas CJ, Rastogi N, Hazbón MH. Phylogenomic analysis of the species of the Mycobacterium tuberculosis complex demonstrates that Mycobacterium africanum, Mycobacterium bovis, Mycobacterium caprae, Mycobacterium microti and Mycobacterium pinnipedii are later heterotypic synonyms of Mycobacterium tuberculosis. Int J Syst Evol Microbiol. 2018;68(1):324-32. https://doi.org/10.1099/ijsem.0.002507.

37. Kim S-Y, Shin SH, Moon SM, Yang B, Kim H, Kwon OJ, et al. Distribution and clinical significance of Mycobacterium avium complex species isolated from respiratory specimens. Diag Microb Infect Dis. 2017;88(2):125-37. https://doi. org/10.1016/j.diagmicrobio.2017.02.017.

38. Dumas E, Boritsch EC, Vandenbogaert $M$, de la Vega RCR, Thiberge J-M, Caro $V$, et al. Mycobacterial pan-genome analysis suggests important role of plasmids in the radiation of type VII secretion systems. Genome Biol Evol. 2016;8(2):387-402. https://doi.org/10.1093/gbe/evw001.

39. Daley $\mathrm{CL}$, laccarino JM Jr, Lange C, Cambau E, Wallace RJ, Andrejak C, et al. Treatment of nontuberculous mycobacterial pulmonary disease: an official ATS/ERS/ESCMID/IDSA clinical practice guideline: executive summary. Eur Respit J. 2020;71:e1-36.

40. Griffith DE, Aksamit T, Brown-Elliott BA, Catanzaro A, Daley C, Gordin F, et al. An official ATS/IDSA statement: diagnosis, treatment, and prevention of 
nontuberculous mycobacterial diseases. Am J Respir Crit Care Med. 2007; 175(4):367-416. https://doi.org/10.1164/rccm.200604-571ST.

41. Maekura R, Okuda Y, Hirotani A, Kitada S, Hiraga T, Yoshimura K, et al. Clinical and prognostic importance of serotyping Mycobacterium aviumMycobacterium intracellulare complex isolates in human immunodeficiency virus-negative patients. J Clin Microbiol. 2005;43(7):3150-8. https://doi.org/1 0.1128/JCM.43.7.3150-3158.2005.

42. Pandey AK, Sassetti CM. Mycobacterial persistence requires the utilization of host cholesterol. Proc Natl Acad Sci U S A. 2008;105(11):4376-80. https://doi. org/10.1073/pnas.0711159105.

43. Forrellad MA, McNeil M, Santangelo Mde L, Blanco FC, García E, Klepp LI, et al. Role of the Mce1 transporter in the lipid homeostasis of Mycobacterium tuberculosis. Tuberculosis(Edinb). 2014;94:170-7.

44. Tateishi Y, Minato Y, Baughn AD, Ohnishi H, Nishiyama A, Ozeki Y, et al. Genome-wide identification of essential genes in Mycobacterium intracellulare by transposon sequencing - implication for metabolic remodeling. Sci Rep. 2020;10(1):5449. https://doi.org/10.1038/s41598-02062287-2.

45. Kim B-J, Hong S-H, Kook Y-H, Kim B-J. Molecular evidence of lateral gene transfer in rpoB gene of Mycobacterium yongonense strains via multlilocus sequence analysis. PLoS One. 2013;8(1):e51846. https://doi.org/10.1371/ journal.pone.0051846.

46. Fedrizzi T, Meehan CJ, Grottola A, Giacobazzi E, Fregni Serpini G, Tagliazucchi S, et al. Genomic characterization of nontuberculous mycobacteria. Sci Rep. 2017;7(1):45258. https://doi.org/10.1038/srep45258.

47. Stinear TP, Seemann T, Harrison PF, Jenkin GA, Davies JK, Johnson PD, et al. Insights from the complete genome sequence of Mycobacterium marinum on the evolution of Mycobacterium tuberculosis. Genome Res. 2008;18(5): 729-41. https://doi.org/10.1101/gr.075069.107.

48. van Helden P, Victor T, Warren $R$, van Helden E. Isolation of DNA from Mycobacterium tuberculosis. In Parish T, Stoker NG, editors. Mycobacterium tuberculosis Protocols, Humana Press; 2001. p 19-30.

49. Tanizawa Y, Fujisawa T, Nakamura Y. DFAST: a flexible prokaryotic genome annotation pipeline for faster genome publication. Bioinformatics. 2018; 34(6):1037-9. https://doi.org/10.1093/bioinformatics/btx713.

\section{Publisher's Note}

Springer Nature remains neutral with regard to jurisdictional claims in published maps and institutional affiliations.

Ready to submit your research? Choose BMC and benefit from:

- fast, convenient online submission

- thorough peer review by experienced researchers in your field

- rapid publication on acceptance

- support for research data, including large and complex data types

- gold Open Access which fosters wider collaboration and increased citations

- maximum visibility for your research: over $100 \mathrm{M}$ website views per year

At $\mathrm{BMC}$, research is always in progress.

Learn more biomedcentral.com/submissions 